\title{
Analysis of the Interaction between THz Waves and Low Cost Plasma Detectors for the Development of Stand-Off Imaging Systems
}

\author{
J. P. Pavia ${ }^{1,2, *}$, M. A. Ribeiro ${ }^{1,2}$, C. Kusoglu-Sarikaya ${ }^{3}$, H. Altan ${ }^{3}$, Demiral Akbar ${ }^{3}$ and N. Souto ${ }^{1,2}$ \\ ${ }^{1}$ University Institute of Lisbon - ISCTE-IUL, Av. das Forças Armadas, 1649-026 Lisbon, Portugal \\ ${ }^{2}$ Instituto de Telecomunicações, Av. Rovisco Pais, 1049-001 Lisbon, Portugal \\ ${ }^{3}$ Department of Physics, Middle East Technical University, 06800, Ankara, Turkey \\ *Joao_Pedro_Pavia@iscte-iul.pt
}

\begin{abstract}
Over the past few years, the Terahertz (THz) band has been seen as quite promising in fields of spectroscopy and imaging. Most of the current manufacturing methods for $\mathrm{THz}$ emission and detection devices are limited due to the associated cost and complexity, therefore alternative ways have been sought driven by the availability of new technologies. From recent research in the field of imaging, commercially available Glow Discharge Detectors (GDDs) have been found to be an interesting solution because of their low cost and efficiency in detecting electromagnetic (EM) radiation from microwave to higher frequencies of the electromagnetic spectrum. Several studies have been carried out in the scope of millimeter/sub-millimeter waves to understand how its detection mechanism changes for higher frequencies and how the mechanisms of plasma generation influence the device's performance. In this paper, we aim to study the interaction between $\mathrm{THz}$ waves and the structure of a GDD, namely, its reflection, transmission and losses. Numerical modelling of electromagnetics, by solving the Maxwell's equations, has been undertaken for this type of device using different gas pressures of the lamp. Our results show that these devices are not optimized for the THz domain.
\end{abstract}

Keywords - Glow discharge detectors (GDDs); terahertz (THz); imaging systems.

\section{INTRODUCTION}

Research within the field of terahertz $(\mathrm{THz})$ has grown rapidly over the past few years and, since then, there has been an increasing demand for high quality electronic and optoelectronic components [1]. Nevertheless, these components cannot be easily manufactured from natural materials because they usually have no resonant response in the $\mathrm{THz}$ frequency region and so the manufacture of artificial materials is an increasingly relevant topic.

Over the last few decades, multiple techniques and imaging systems have been developed in the field of millimeter wave (mmWave)/THz due to the possibility that they offer to detect and visualize hidden objects or dangerous materials at stand-off distances. However, mmWave and $\mathrm{THz}$ wave detection/generation technologies are very costly and the systems that have been commercially developed are not robust enough to be implemented in several environments.

In 1970s, a new area of research, which consisted in the use of Glow Discharge Detectors (GDDs) for the detection of microwaves and millimeter waves, began [2]. By forming a discharge in a gas kept at high pressure between two electrodes, the lamp generates light and a plasma is formed inside the device, which makes it sensitive, and consequently, able to detect electromagnetic radiation ranging from microwave to $\mathrm{mmWave} / \mathrm{THz}$ waves. Commercial indicator lamps filled with a gas like Neon were used by Farhat and Kopeika to demonstrate the capabilities of electromagnetic radiation detection of these low-cost devices [3]. Since then, several studies have been carried out to understand the mechanism of operation of these devices and how it is possible to maximize their sensitivity, dynamic range and responsivity, especially in the mmWave band. Although these studies were conducted in ranges close to $\mathrm{THz}$, they allow us to understand how the parameters described above are affected as we advance the frequency for each type of device under analysis [4].

The GDDs present a very interesting potential of application with focus on $\mathrm{THz}$ imaging, since it is possible to use these devices for the construction of focal-plane arrays (FPA), which are a fundamental part of the acquisition of video and image with greater sharpness and quality by the imaging devices [5]. Therefore, it is necessary to understand how these devices will behave in the $\mathrm{THz}$ band and how the characteristics related to the design and the specifications contribute to the detection of radiation.

In this paper, we intend to simulate a GDD structure and analyze the interaction between $\mathrm{THz}$ radiation and these devices. The paper is organized as follows: In section II, we present an overview about the GDD structure simulation design. Numerical simulations are presented in section III. The results obtained allow us to determine the characteristic curves of the GDD for its reflection, transmission and losses as a function of gas pressure. Finally, the conclusions are outlined in section IV.

\section{GDD STRUCTURE SimULATION DESIGN}

In order to use the GDDs in $\mathrm{THz}$ radiation detection applications, we must understand several topics from the generation of the plasma to the interaction between the EM waves and the structure of these devices.

There are several methods to generate plasma. The most common ones can be characterized by applying a strong electric field in a medium filled with gases (e.g. considering a 
strong gradient direct current (DC) voltage difference that is applied between two electrodes) or by coupling a radio frequency (RF) signal via an inductor that is inside the gaseous medium. At last, plasma can be also generated by a strong EM signal in a whole range of EM spectrum focused inside of that gaseous medium [4]

Considering only the electrode structures, we know that their interaction with EM waves is similar to that described in frequency selective surfaces. It was mentioned in [6] that the transmittance and the reflectance through a metallic structure is highly dependent on the frequency, being also influenced by other aspects related to the propagation direction of the waves from the source and the movement of the electrons. From this interaction, the electrons in metals will absorb and re-radiate some wavelengths with higher efficiency than others [6]. However, the interaction of EM waves between the electrodes and plasma is more complex to describe when compared to basic scenarios.

$\mathrm{THz}$ pulses passing between the electrodes undergo a time domain diffraction and a spatial frequency domain diffraction. As a result of this scattering, it is not possible to determine the frequencies that are propagating through the structure and those that cannot progress. In order to simulate the whole GDD structure (Fig.1), especially at $\mathrm{mm} / \mathrm{THz}$ wavelengths, it is necessary to choose a suitable substructure and on which numerical analysis can be performed [7]:

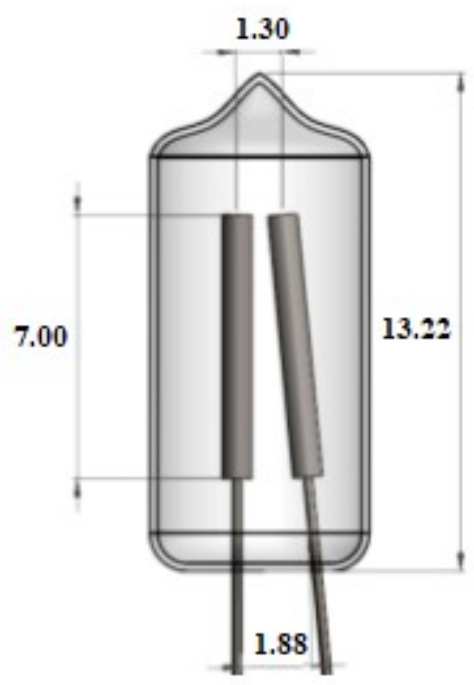

Fig. 1. Technical drawing of a glow discharge detector (GDD), in which all dimensions are given in millimeters. The diameter of each electrode is 0.79 $\mathrm{mm}$, and outer diameter of the glass envelope is $5.82 \mathrm{~mm} \mathrm{[7].}$

The EM wave propagation in a cold, collisional, isotropic plasma with no background magnetic field can be characterized by the relative permittivity given by [8]

$$
\varepsilon_{r}(x)=1-\frac{\omega_{p}{ }^{2}(x)}{\omega\left(\omega+i v_{e}(x)\right)},
$$

where $x$ denotes the electron's position between electrodes, $\omega$ is the angular frequency of the propagating electromagnetic wave, $\omega_{p}$ is the plasma frequency and $v_{e}$ is the electron's effective collision frequency. The last two parameters, $\omega_{p}$ and $v_{e}$ were obtained through parallel 1d3v PIC/MCC simulation and the relative permittivity was calculated based on the position of the electron between the electrodes [9].

Plasma also has a dielectric loss constant, which quantifies the inherent dissipation from electromagnetic energy. It can be parameterized in terms of the angle of loss, $\delta$, or the loss tangent [8]:

$$
\tan (\delta)=\frac{\operatorname{Im}\left(\varepsilon_{r}\right)}{\operatorname{Re}\left(\varepsilon_{r}\right)}
$$

By knowing these two parameters, $\varepsilon_{r}$ and $\delta$, the plasma can be characterized as a material for electromagnetic simulation purposes. The design of Fig. 2 was considered to model the problem stated before.
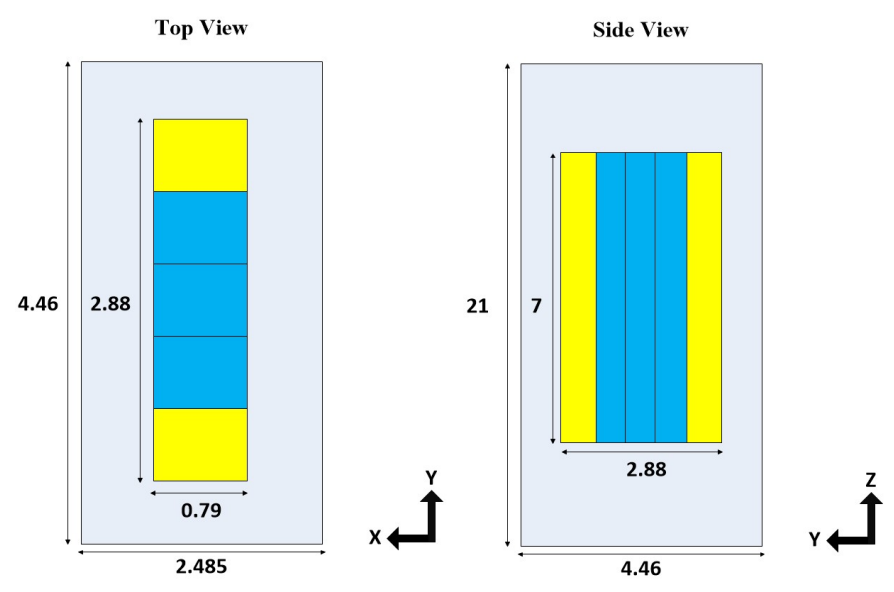

Fig. 2. Design of the structure showing the electrode structure and plasma medium to be developed for THz-plasma interaction measurements on a simulation environment, in which all dimensions are given in millimeters.

The electrode material in yellow is selected as an ideal conductive material and the width of the plasma medium indicated in blue is limited by the thickness of the electrode in this design $(0.79 \mathrm{~mm})$. In this figure, it is also possible to see three boxes that model the plasma for each position of the electron. In general, the larger the number of boxes, the more refined the results of model simulations are, since there is a greater amount of data regarding the propagation of electromagnetic waves in the plasma formed between the electrodes.

\section{NUMERICAL RESULTS}

The results of our numerical simulations using the parameters outlined in the previous section are shown in the following figures for a target frequency of $342 \mathrm{GHz}$ and a frequency sweep from 300 to $380 \mathrm{GHz}$. Fig. 3 shows the numerical simulation, using the Finite Element Method, for the reflection and transmission of the GDD as a function of frequency for various degrees of pressure of the gases inside the structure. In the simulations, we considered a similar methodology to the one that is used in the simulation of dipole antennas, since the GDD has a dipole antenna-like structure 
[3]. We imposed the necessary radiation boundary condition and two excitations defined as wave ports. This model is clearly an approximation of the real problem, but it was used to avoid excessively high complexity for the simulation.

The gas pressure inside the device generally does not influence the reflection of the device. However, above the target frequency, an increase in reflection is observed, in which the magnitude of the reflection is influenced by the gas pressure as shown in Fig. 3:

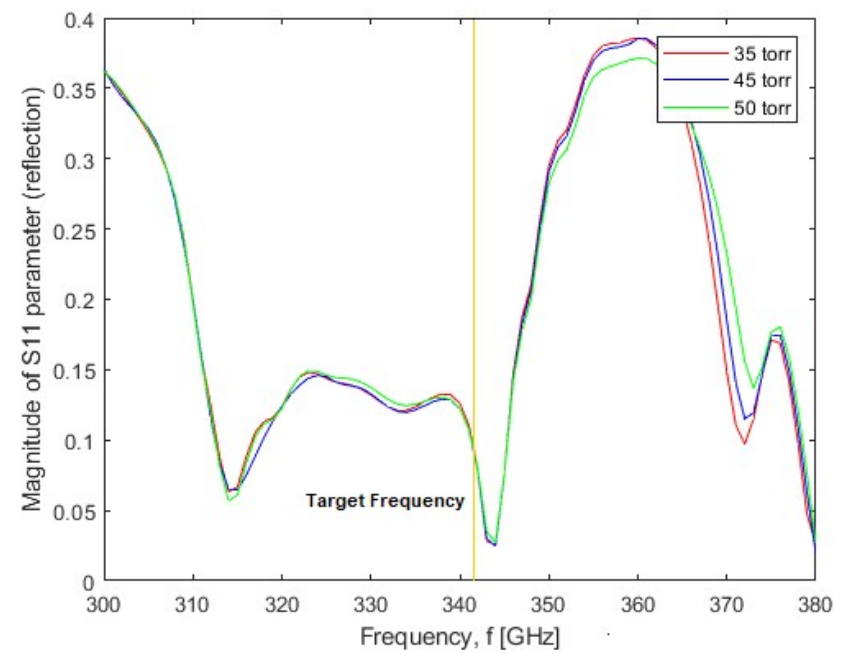

Fig. 3. Reflection in the frequency domain for 35, 45 and 50 torr of pressure.

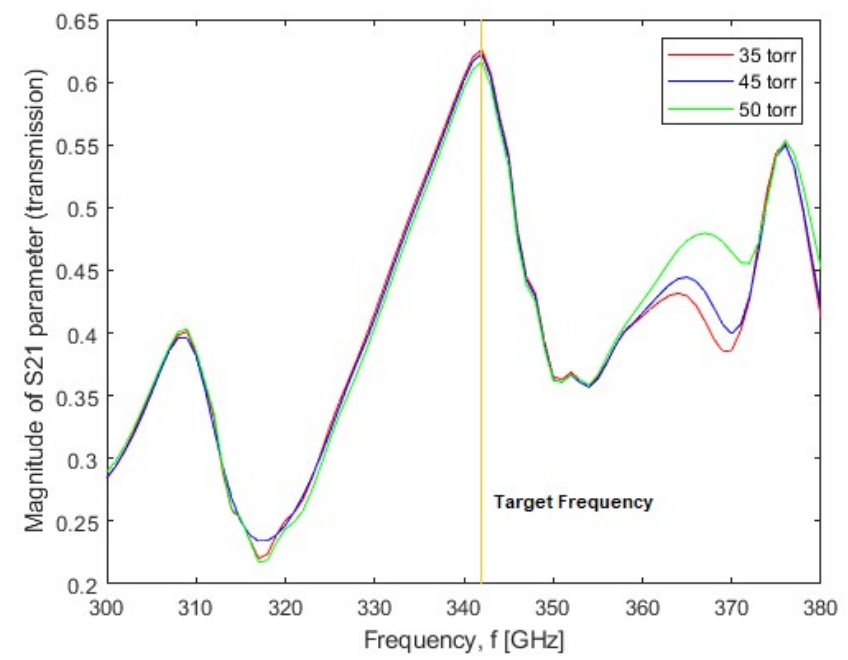

Fig. 4. Transmission in the frequency domain for 35,45 and 50 torr of pressure.

Analyzing Fig. 4, it is observed that as the gas pressure increases the transmission suffers little variation with frequency. The structure has a reasonable dynamic range for the target frequency, in which the maximum transmission takes place, also observing that it is larger in the frequency range between 328 and $350 \mathrm{GHz}$. However, for frequencies above $350 \mathrm{GHz}$, the transmission becomes more sensitive to pressure.

Through the S parameters, we can also characterize these devices in relation to the losses they present, since this is a common characteristic of any passive device. Therefore, we can study the variation of the Power Scattering Ratio (PS) that the structure undergoes in the frequency domain, which is defined as

$$
P S=\left|S_{11}\right|^{2}+\left|S_{21}\right|^{2}
$$

This ratio shows the amount of electric power consumed by the device. The smaller the result for the calculation $(0 \leq P S \leq 1)$, the greater the loss, as shown in Fig. 5 for each gas pressure.

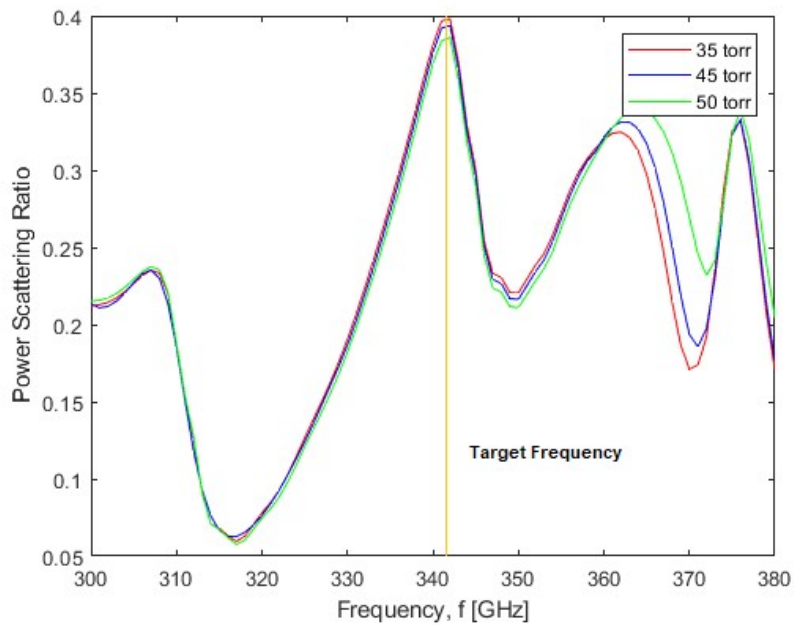

Fig. 5. Power scattering ratio in the frequency domain for 35, 45 and 50 torr of pressure.

For the target frequency, the structure has the lowest loss level when compared to the remaining frequencies of the selected range under analysis. In fact, the smaller the pressure, the greater the PS. However, this trend is reversed for frequencies between 350 and $375 \mathrm{GHz}$.

In an ideal scenario, we would expect a transmission of energy close to $100 \%$. However, this is only possible if we have an exact match between the source impedance, the characteristic impedance of the transmission line and all its connectors, and the load's impedance. Mismatched impedances are a common issue in real scenarios, in which they may cause some of the power to be reflected back toward the source. This phenomenon gave rise to destructive interference, leading to peaks and valleys in the voltage along the line [10]. The voltage standing wave ratio (VSWR) measures these voltage variances an it is expressed as

$$
V S W R=\frac{1+\left|S_{11}\right|}{1-\left|S_{11}\right|} .
$$

The smaller the VSWR, the better the matching of the impedances and the greater the power to be delivered. The minimum VSWR is 1.0, which means that no power is reflected (ideal scenario). For the considered frequency range of the previous figures, we measured this ratio for each gas pressure. The results are shown in Fig. 6: 


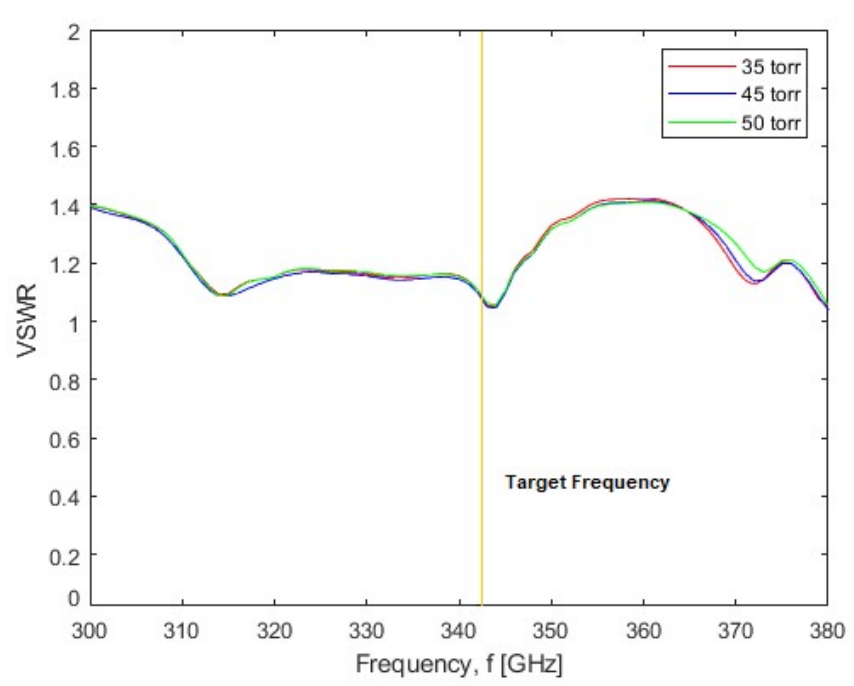

Fig. 6. VSWR in the frequency domain for 35,45 and 50 torr of pressure.

Analyzing this figure, only minor difference of VSWR are observed between the different pressures except for frequencies above $360 \mathrm{GHz}$. In general, the minimum VSWR rounds 1.04 and the maximum rounds 1.4, which are considered as reasonable values. As a final remark and independently of the measurement under analysis, we can state that these structures are not optimized for the $\mathrm{THz}$ domain, so it is necessary to focus on their design to optimize the response of these devices.

\section{CONCLUSIONS}

According to the simulation results, we concluded that the gas pressure inside the GDDs influences the behavior of these devices in terms of the reflection, transmission and losses of incident wave energy in small frequency ranges.

The observed behavior of the GDD suggests a frequency dependent interaction that cannot be explained by the interaction of the EM radiation and the plasma itself. In fact, this frequency dependent transmission suggests that the structure of the device plays an important role on the propagation of the $\mathrm{THz}$ waves.

In this simulation study, the discharge structures of a commercial GDD with different pressures were designed to be used in the THz domain. However, our data suggests that the most efficient (optimal) structures can be determined by designing the structures of the electrode.

\section{ACKNOWLEDGMENT}

The authors acknowledge the financial support by Fundação para a Ciência e a Tecnologia under the project TUBITAK/0002/2014.

\section{REFERENCES}

[1] B. Ferguson and X. Zhang, "Materials for terahertz science and technology," Nature Materials, vol. 1, n 1, pp. 26-33, 2002.

[2] A. Abramovich, N. Kopeika, D. Rozban and E. Farber, "Inexpensive detector for terahertz imaging", Applied Optics, vol. 46, no. 29, p. 7207, 2007.

[3] N. Farhat and N. Kopeika, "A low-cost millimeter-wave glow-discharge detector", Proceedings of the IEEE, vol. 60, no. 6, pp. 759-760, 1972.

[4] N. Kopeika, "Glow Discharge Detection of Long Wavelength Electromagnetic Radiation: Cascade Ionization Process Internal Signal Gain and Temporal and Spectral Response Properties", IEEE Transactions on Plasma Science, vol. 6, no. 2, pp. 139-157, 1978.

[5] D. Rozban, N. Kopeika, A. Abramovich and E. Farber, "Terahertz detection mechanism of inexpensive sensitive glow discharge detectors", Journal of Applied Physics, vol. 103, no. 9, p. 093306, 2008.

[6] J. S. Fernandez, "Frequency selective surfaces for Terahertz applications, PhD Thesis," The University of Edinburgh, 2012.

[7] K. Cinar, H. Bozaci and H. Altan, "Characterization of a Glow Discharge Detector With Terahertz Time Domain Spectroscopy", IEEE Sensors Journal, vol. 13, no. 7, pp. 2643-2647, 2013.

[8] J. Nicolini and J. Bergmann, "Finite-Difference Time Domain Techniques Applied to Electromagnetic Wave Interactions with Inhomogeneous Plasma Structures", International Journal of Antennas and Propagation, vol. 2018, pp. 1-20, 2018.

[9] C. Kusoglu-Sarikaya, H. Altan and D. Akbar, "Parallel 1d3v Particle in Cell/Monte Carlo Collision (PIC/MCC) Simulation of a Glow Discharge Millimeter Wave Detector", Proceedings of the 6th International Conference on Photonics, Optics and Laser Technology, 2018.

[10] D. Pozar, Microwave Engineering, John Wiley \& Sons, 2012. 